\title{
Intestinal flora alterations in patients with ulcerative colitis and their association with inflammation
}

\author{
ZHI FENG DAI ${ }^{1 *}, X^{*}$ YUAN MA ${ }^{2 *}$, RUI LIN YANG ${ }^{1 *}$, HUI CHAO WANG ${ }^{3}$, DAN DAN XU $^{4}$, JING NAN YANG $^{1}$, \\ XIAO BING GUO ${ }^{5}$, SHUANG SHUANG MENG ${ }^{1}$, RUI XU ${ }^{1}$, YU XIA LI $^{1}$, YAO XU $^{1}, \mathrm{KUN} \mathrm{LI}^{6}$ and XU HONG LIN ${ }^{1}$ \\ ${ }^{1}$ Department of Clinical Laboratory, Translational Medicine Center, Huaihe Hospital of Henan University, \\ Kaifeng, Henan 475000; ${ }^{2}$ Department of Gastroenterology, People's Hospital of Xuchang, Xuchang, Henan 461000; \\ ${ }^{3}$ Department of Nephrology, The First Affiliated Hospital of Henan University, Kaifeng, Henan 475000; \\ ${ }^{4}$ Department of Dermatology, Huaihe Hospital of Henan University, Kaifeng, Henan 475000; \\ ${ }^{5}$ Department of Clinical Laboratory, The First Affiliated Hospital of Zhengzhou University, \\ Zhengzhou, Henan 450002; ${ }^{6}$ Department of Pathophysiology, Institute of Digestive Disease, \\ Tongji University School of Medicine, Shanghai 200092, P.R. China
}

Received December 4, 2018; Accepted March 17, 2021

DOI: $10.3892 / \mathrm{etm} .2021 .10757$

\begin{abstract}
Ulcerative colitis (UC), which is a type of inflammatory bowel disease, is a chronic intestinal disorder of multifactorial etiology. Numerous studies have indicated an association between UC and intestinal bacteria. However, a limited number of studies regarding the expression of interleukin-17 (IL-17) and interleukin-23 (IL-23) in association with intestinal bacteria have been performed. The aim of the current study was to investigate the gut microbiota alterations in patients with UC, at a number of taxonomic levels, and their relationship with intestinal inflammation by analyzing the protein expression of IL-17 and IL-23. Specimens were collected from 10 healthy controls and 16 patients with UC. A histological examination was performed in colonic tissues, IL-17 and IL-23 protein expression was detected by immunohistochemistry, fecal samples were sequenced using 16S rDNA sequencing and bioinformatics analysis was performed. The UC group exhibited an increased histological score $(\mathrm{P}<0.01)$ and upregulated IL-17 and IL-23 expression $(\mathrm{P}<0.01)$. At the order level, the bacterial diversity of the UC group was decreased. $\beta$-diversity analyses, including principal component analysis, principal coordinate analysis and non-metric multidimensional scaling, demonstrated that the two groups of samples were
\end{abstract}

Correspondence to: $\mathrm{Dr} \mathrm{Xu}$ Hong Lin, Department of Clinical Laboratory, Translational Medicine Center, Huaihe Hospital of Henan University, 115 Ximen Street, Kaifeng, Henan 475000, P.R. China

E-mail: 10220017@vip.henu.edu.cn

*Contributed equally

Key words: ulcerative colitis, intestinal bacteria, inflammation, interleukin-17, interleukin-23 separated into two taxonomic categories, as distinct variations were observed in the analysis of group differences $(\mathrm{P}=0.001)$. Regarding the differences in species composition between the groups, Enterococcus was indicated to be the species with the greatest difference in abundance compared with the healthy control group $(\mathrm{P}<0.01)$, followed by Lactobacillus $(\mathrm{P}<0.05)$, Escherichia-Shigella $(\mathrm{P}<0.05)$, Bifidobacterium and Bacteroides. In addition, the average optical density of IL-17 was positively correlated with the histological score $(\rho=0.669$; $\mathrm{P}=0.035)$, Enterococcus $(\mathrm{r}=0.843 ; \mathrm{P}<0.001)$, Lactobacillus $(\mathrm{r}=0.737 ; \mathrm{P}=0.001)$, Bifidobacterium $(\mathrm{r}=0.773 ; \mathrm{P}<0.001)$ and Escherichia-Shigella $(\mathrm{r}=0.663 ; \mathrm{P}=0.005)$, and the average optical density of IL-23 was positively correlated with the histological score $(\rho=0.733 ; \mathrm{P}=0.016)$, Enterococcus $(\mathrm{r}=0.771$; $\mathrm{P}<0.001)$, Lactobacillus $(\mathrm{r}=0.566 ; \mathrm{P}=0.022)$, Bifidobacterium $(\mathrm{r}=0.517 ; \mathrm{P}=0.041)$ and Escherichia-Shigella $(\mathrm{r}=0.613$; $\mathrm{P}=0.012$ ). The results of the present study indicated that the intestinal microbiota of patients with UC differed from that of healthy controls at multiple taxonomic levels. The alterations of the intestinal microflora were closely associated with the degree of inflammation. The IL-23/IL-17 axis, as a key factor in the development of UC, maybe associated with the alterations of intestinal microflora. The interaction between intestinal microflora and the IL-23/IL-17 axis may serve an important role in the pathogenesis of $\mathrm{UC}$.

\section{Introduction}

Ulcerative colitis (UC), which is a type of inflammatory bowel disease (IBD), is a chronic intestinal disorder of multifactorial etiology $(1,2)$. The principal outcome of UC is the development of colorectal cancer $(3,4)$.

The pathogenesis of IBD is not fully understood. It is believed that chronic inflammation and immune response disorders are the key pathological features in UC, whereas an imbalance in gut microbiota is a key factor leading to inflammation and abnormal immune response, thereby contributing 
to disease initiation and progression (5). Under a normal physiological state, the intestinal flora remains stable and interacts with the host, serving an important role in nutrient absorption, the prevention of pathogen invasion and the maintenance of normal immune function (6). As the microbiota is an important factor that is associated with the maturation of the early immune system, it effectively establishes an interaction with the host and is the principal factor leading to chronic inflammatory disorders, such as UC (7). Previous observations from clinical and experimental studies have indicated that an intestinal bacteria imbalance is associated with disease initiation and progression in UC $(8,9)$. The alterations in the characteristics of the intestinal flora of patients with IBD include variations in the total number of mucosal bacteria and diversities in the composition of the flora early in the disease (10).

A previous study reported that the amount of total bacteria is decreased in patients with active UC, especially the balance of Staphylococci/Bacilli, which is characterized by an increased abundance of Staphylococci and a decreased abundance of Bacilli. Subsequently, UC was attributed to the alterations in the abundance of several bacterial species (11). In our previous study (12), the abundance of different types of bacteria was examined using PCR, and it was revealed that UC was attributed to the alterations in the abundance of several bacterial species.

A recent study revealed that the manifestation of dysbiosis in patients with UC is characterized by a decrease in bacterial diversity, and this study concluded that an alteration in the diversity and composition of the gut microbiome, rather than the presence of specific pathogens, is likely to serve a critical role in disease progression (11). Furthermore, the abundance of invasive bacteria types was indicated to increase, and that of protective bacteria types was revealed to decrease in patients with UC (13). For example, it was demonstrated that the increased abundance of Enterobacteriaceae, Pasteurellaceae, Veillonellaceae and Fusobacteriaceae, and the decreased abundance of Erysipelotrichales, Bacteroidales and Clostridiales, were associated with UC disease status (14). Another study has indicated that in patients with both Crohn's disease (CD) and UC, a decreased biodiversity, a reduced proportion of Firmicutes and an increased abundance of Gammaproteobacteria were observed when compared with healthy controls (15). In addition, it is widely believed that the gut microbiota of patients with active UC is different from that of healthy controls, whereas to the best of our knowledge, no evidence for a difference between remission and control groups exists. Hence, the current study aimed to assess whether gut microbiota may be a potential target for controlling UC progression.

Currently, there is little evidence to support the alterations in intestinal aerobes and common anaerobes in patients with UC, owing to the differences in the disease course, stage, treatment, analysis method and microbiota complexity. Additionally, their specific role in the occurrence of the host disease has not yet been fully elucidated. Furthermore, to the best of our knowledge, a relationship between microbiota alterations and mucosal damage has not yet been demonstrated. The majority of the studies on intestinal inflammation have focused on the association between interleukins and the immune system, which demonstrated that interleukin-17 (IL-17) and interleukin-23 (IL-23) serve a critical role in intestinal inflammation and exhibit a typical positive correlation with UC (16-18). In the present study, the differences in intestinal flora between patients with UC and healthy controls were compared, and the relationship between microbiota alterations and inflammation was explored, which provided novel insights that may be used for the diagnosis and treatment of UC.

\section{Materials and methods}

Patient recruitment. A total of 16 patients with UC (age range, 40-60 years, median age, 53 years; 7 male and 9 female) were recruited at Huaihe Hospital of Henan University (Kaifeng, China) from July 2016 to June 2017. Patients with different degrees of abdominal pain, diarrhea and mucopurulent bloody stool, who were diagnosed with UC by fibro-colonoscopy and routine pathological examination [meeting the diagnostic criteria published in the Consensus on the Standards for the Diagnosis and Cure of Inflammatory Bowel Diseases in China (19)], were included in the current study according to the Montreal standard for the evaluation of clinical performance (20). A total of 10 healthy control volunteers (age range, 35-60 years old, median age, 46 years old; 4 male and 6 female) who had not received antibiotics in the previous three months, were recruited at Huaihe Hospital of Henan University (Kaifeng, China) from July 2016 to June 2017. The healthy controls displayed no evidence of active pathology. Written informed consent was obtained from all patients prior to the procedure. Ethical approval was obtained from the Medical Ethics Committee of Huaihe Hospital of Henan University (Kaifeng, China). The patients were excluded from the present study if at least one of the following criteria were met: i) Received antibiotics or probiotics within 4 weeks before specimen collection; ii) diagnosed with infective enteritis, such as bacterial dysentery, intestinal tuberculosis, schistosomiasis, Crohn's disease, ischaemic enteritis, radiation enteritis, irritable bowel syndrome or colon carcinoma, via fibro-colonoscopy; iii) suffered from coronary heart disease, hypertensive disease, diabetes, active pulmonary tuberculosis or peptic ulcers.

Histological evaluation. Colon tissues were fixed in $4 \%$ paraformaldehyde at room temperature overnight. Paraffin-embedded colon tissues were cut into 5- $\mu$ m-thick sections for hematoxylin and eosin (H\&E) staining, the slices were stained at room temperature for $5 \mathrm{~min}$ with hematoxylin and $1 \mathrm{~min}$ with eosin. The histological index was estimated based on the histological severity of colitis. The pathological sections, which were stained with $\mathrm{H} \& \mathrm{E}$, were observed under a light microscope (magnification, x200) and were scored independently by two pathologists via the blind method. According to the specific scoring criteria (21), inflammatory cell infiltration ( 0 point, sporadic inflammatory cells in lamina propria; 1 point, number of inflammatory cells in lamina propria increased; 2 points, number of inflammatory cells extended to the submucosa; and 3 points, infiltration of inflammatory cells into the intestinal wall) and tissue damage (0 point, no mucosal injury; 1 point, lymphoepithelial injury; 2 points, surface mucosal erosion or focal ulcer; and 3 points, extensive mucosal injury and structure extending to deeper intestinal wall) were combined. The details are presented in Table I. The two sub-scores were added and the combined histological score ranged from 0 (no alterations) to 6 (highest score with extensive cell infiltration and tissue damage). 
Table I. Scores of histology activity.

\begin{tabular}{lc}
\hline Property & Score \\
\hline $\begin{array}{l}\text { Inflammatory cell infiltration } \\
\text { Sporadic inflammatory cells in lamina propria }\end{array}$ & 0 \\
$\begin{array}{l}\text { Number of inflammatory cells in lamina propria } \\
\text { increased }\end{array}$ & 1 \\
$\begin{array}{l}\text { Number of inflammatory cells extended to the } \\
\text { submucosa }\end{array}$ & 2 \\
$\begin{array}{l}\text { Infiltration of inflammatory cells into the intestinal } \\
\text { wall }\end{array}$ & 3 \\
$\begin{array}{l}\text { Tissue damage } \\
\text { No mucosal injury }\end{array}$ & \\
$\begin{array}{l}\text { Lymphoepithelial injury } \\
\text { Surface mucosal erosion or focal ulcer }\end{array}$ & 0 \\
Extensive mucosal injury and structure extending \\
to deeper intestinal wall
\end{tabular}

The histological score ranged from 0 (unchanged) to 6 (extensive cell infiltration and tissue damage).

Immunohistochemistry for $I L-17$ and IL-23 protein. Colon tissues were fixed in $4 \%$ paraformaldehyde at room temperature overnight. Paraffin-embedded colon tissues were sliced into sections $\left(5-\mu \mathrm{m}\right.$ thick), and were subjected to dewaxing $\left(60^{\circ} \mathrm{C}\right.$ for $2 \mathrm{~h}$ ), followed by soaking in dimethylbenzene twice for 15 min, hydration (ethyl alcohol was used twice, 95\% alcohol and $80 \%$ alcohol once, all for $1 \mathrm{~min}$ respectively; $70 \%$ alcohol once for $2 \mathrm{~min}$. All alcohols used were analytically pure), antigen retrieval and washing with $\mathrm{PBS} .3 \% \mathrm{H}_{2} \mathrm{O}_{2}$ was used to block the endogenous peroxidase for $10 \mathrm{~min}$ at room temperature, and the slides were incubated with anti-IL-17 (1:50; cat. no. ab79056; Abcam) or anti-IL-23 (1:200; cat. no. ab45420; Abcam) antibody dissolved in blocking solution (QuickBlock ${ }^{\mathrm{TM}}$; Beyotime Institute of Biotechnology) at $4^{\circ} \mathrm{C}$ overnight. After incubation, the slides were washed with PBS and incubated with an HRP-labelled polymer system (used neat; cat. no. SP-0023; Beijing Boaosen Biotechnology Co., Ltd.) at $37^{\circ} \mathrm{C}$ for $15 \mathrm{~min}$, followed by an incubation with 3,3-diaminobenzidine detection reagent at room temperature for $5 \mathrm{~min}$, and finally observed under light microscope with a magnification of $\mathrm{x} 200$. The semi-quantitative expression of each protein was analyzed by Image-Pro Plus software v.6.0 (Media Cybernetics, Inc.).

DNA extraction. Fresh stool specimen from the control and UC groups were collected in a sterile container, and then stored in the refrigerator at $-80^{\circ} \mathrm{C}$ within $30 \mathrm{~min}$. A total of 26 fecal bacterial DNA samples were extracted using a fecal nucleic acid extraction kit (cat. no. DP328-02; Tiangen Biotech Co., Ltd.), with additional proteinase $\mathrm{K}$ treatment at $70^{\circ} \mathrm{C}$ for $10 \mathrm{~min}$ to ensure adequate bacterial cell lysis. The DNA extraction for all included biospecimens was performed at a single center by the same person using an identical protocol and the same extraction kit for all samples.

Amplicon sequencing and bioinformatics analysis. The MetaVx $^{\mathrm{TM}}$ library preparation and Illumina MiSeq next generation sequencing (NGS) were performed at Genewiz, Inc.. The DNA samples were quantified using a Qubit 2.0 Fluorometer (Thermo Fisher Scientific, Inc.). In total, 30-50 ng DNA was used to generate amplicons using a MetaVx ${ }^{\mathrm{TM}}$ Library Preparation kit (Genewiz, Inc.). The V3 and V4 hypervariable regions of prokaryotic $16 \mathrm{~S}$ rRNA were selected for the generation of amplicons and subsequent taxonomic analysis. Genewiz, Inc. designed a panel of proprietary primers targeting relatively conserved regions bordering the $\mathrm{V} 3$ and V4 hypervariable regions of the 16S rRNA of bacteria and archaea. The V3 and V4 regions were amplified using forward primers containing the sequence 5'-CCTACGGRR BGCASCAGKVRVGAAT-3' and reverse primers containing the sequence 5'-GGACTACNVGGGTWTCTAATCC-3'. The first-round PCR products were used as templates for the second-round amplicon enrichment PCR. Indexed adapters were added to the ends of the $16 \mathrm{~S}$ rRNA amplicons to generate indexed libraries ready for the downstream NGS Illumina MiSeq sequencing. The DNA libraries were validated by an Agilent 2100 Bioanalyzer (Agilent Technologies, Inc.) and quantified by a Qubit 2.0 Fluorometer. The DNA libraries were multiplexed and loaded on an Illumina MiSeq instrument according to the manufacturer's instructions (Illumina, Inc.). Sequencing was performed using a $2 \times 300$ paired-end configuration; image analysis and base calling were performed using the MiSeq Control Software v.2.5.0.5 (Illumina, Inc.) on the aforementioned MiSeq instrument.

Data analysis. The QIIME data analysis packagev.1.8.0 (http://bio.cug.edu.cn/qiime/) was used for the 16S rRNA data analysis. The forward and reverse reads were combined and assigned to samples based on the barcode. Raw reads were trimmed using QIIME. Quality filtering on the combined sequences was performed, and sequences that did not fulfil the following criterion were discarded: Sequence length $<20$ nucleotides. Subsequently, the sequences were compared with those of the reference ribosomal database project (RDP) Gold database v.2.2 (http://rdp.cme.msu.edu/clas sifier/classifier.jsp;jsessionid=D5D6C78C6C197C015E 237D0FD7A85246.10.0.0.9) using the UCHIME (http://www. drive5.com/uchime/uchime_download.html) algorithm to detect chimeric sequences, and the chimeric sequences were removed. The remaining sequences were used in the final analysis. The sequences were grouped into operational taxonomic units (OTUs) using the clustering program VSEARCH (v.1.9.6; https://github.com/torognes/vsearch) against the SILVA 119 database (https://www.arb-silva.de/search/), pre-clustered at $97 \%$ sequence identity. The RDP classifier was used to assign taxonomic categories to all OTUs at a confidence threshold of 0.8. The RDP classifier used the SILVA 123 database (https://www.arb-silva.de/search/), which had taxonomic category predictions down to the species level. The sequences were rarefied prior to the calculation of $\alpha$ - and $\beta$-diversity statistics. $\alpha$-diversity indexes were calculated in QIIME from rarefied samples using the Shannon index for diversity (22) and the Chaol index (23) for richness. Rarefaction curve was used to judge whether the sample size was sufficient and to estimate the species richness. $\beta$-diversity was calculated using weighted and unweighted UniFrac distances, and a principal coordinate analysis (PCoA) and a principal 
component analysis (PCA) were performed. The unweighted pair group method with arithmetic mean (UPGMA) was used to build a tree from the $\beta$-diversity distance matrix. Nonmetric Mutidimensional Scaling (NMDS) was used to reflect the differences between the samples by the distance between points. Analysis of similarities (ANOSIM) (24) was confirmed according to Bray-Curtis.

Statistical analysis. The data are presented as the mean \pm standard deviation. To determine the statistical significance, all the data were analyzed with independent-samples t-tests for normally distributed data or Mann-Whitney tests for non-normally distributed data, with analyses performed using SPSS version 13.0 software (SPSS, Inc.). The associations between the average optical density of IL-17/IL-23 and the histopathological scores in the intestinal mucosa were analyzed using Spearman's correlation test, and the associations between the average optical density of IL-17/IL-23 and the abundance of Enterococcus, Lactobacillus, Bacteroides, Bifidobacterium and Escherichia-Shigella, were analyzed using Pearson's correlation test. $\mathrm{P}<0.05$ was considered to indicate a statistically significant difference.

\section{Results}

Histological examination. A histological examination of the colon was performed to validate the degree of inflammation. As illustrated in Fig. 1, the H\&E staining showed that no signs of inflammation were observed in the control group. The epithelial cells of the normal colonic mucosa were intact, and the glands were arranged neatly in proximity. The goblet cells were abundant, with a few neutrophils and lymphocytes being scattered in the lamina propria, and the capillaries did not display a compressed or enlarged appearance. In the UC group, the colonic mucosa was damaged and eroded, the epithelial cells and the lacunae were destroyed, the glands were arranged in a distorted manner, the goblet cells were absent, and a large number of neutrophils and lymphocytes had infiltrated the lamina propria and the myometrium. Capillary hyperemia and dilatation, as well as a formation of multiple ulcers, were observed. In total, an apparent colitis with characteristic ulcers, multiple erosive lesions, a loss of entire crypts in the colon, as well as a marked inflammatory cell infiltration into the colonic submucosa, were observed; thus, the histological score was higher compared with the control group $(\mathrm{P}<0.01)$.

IL-17 and IL-23 expression in colonic tissues. Immunohistochemical analysis showed that IL-17 and IL-23 proteins were weakly expressed in normal colonic mucosal tissues, while they were increased in UC tissues, and were primarily localized in mucosal epithelial cells and lamina propria. The cytoplasm of the mononuclear cells in UC tissues was stained in a brown-yellow color, which indicated positive IL-17 and IL-23 staining, and the average optical density of IL-17 and IL-23 expression was increased in UC compared with the control groups $(\mathrm{P}<0.01$; Fig. 2).

Gut microbiota variation PCoA, PCA and UPGMA clustering analysis. After the unqualified sequences were removed, a total of 3,706,470 raw reads and an average of 142,557 reads per sample were obtained. Following quality filtering of the reads, a total of 1,853,235 high-quality reads were generated, and each fecal sample produced an average of 71,278 high-quality reads. The samples with a low number of high-quality reads $(<3,000)$ were not analyzed.

According to the UniFrac-based PCoA (Fig. 3A), the gut microbiota in both the control and UC groups was divided into two groups. The gut microbiota composition exhibited a high level of variation between the two groups. The principal coordinates 1 and 2 explained 22.05 and $12.47 \%$ of the total composition variation, respectively. The multivariate analysis of variance of the PCoA matrix scores indicated a statistically significant difference between the microbiota of the control and that of the UC group. The UC group was statistically different from the control group, according to the PCA analysis $(\mathrm{P}<0.05$; Fig. 3B). The results of the control group clustering analysis suggested that the samples were similar. The distribution in the UC group was fragmented but was distinct from that of the control group. The intestinal flora composition exhibited multiple cluster directions but was statistically different from that of the control group in total.

According to the UPGMA clustering analysis (Fig. 3C), these two groups were separated into two different tree clusters, and the samples of each group were clustered well together and were separated into two distinct subgroups.

$\alpha$-diversity and rarefaction analysis. Fig. 4A and B revealed that microbial $\alpha$-diversity, which was assessed by the Chao 1 and Shannon indexes, was decreased in the UC group compared with the healthy control group. A rarefaction analysis was performed to cluster all OTUs, which were present in the data. As shown in Fig. 4C, all the rarefaction curves reached a plateau, suggesting that the sequencing depth for all samples captured the bacterial diversity in these communities.

\section{Variations in bacterial community diversity}

Bacterial community composition at the phylum level. Compared with that in the control group, the abundance of Bacteroidetes was decreased, the abundance of Proteobacteria and Actinobacteria was increased, and the abundance of Firmicutes was slightly increased in the UC group (Fig. 5A).

Bacterial community composition at the class/order level. At the class level, the abundance of Bacilli, Gammaproteobacteria and Actinobacteria in the UC group was increased, the abundance of Clostridia and Bacteroidia was decreased, and that of Negativicutes and Coriobacteriia exhibited a slight decrease (Fig. 5B).

At the order level, the abundance of Lactobacillales and Enterobacteriales was increased in the UC group compared with that in the control group, the abundance of Bifidobacteriales and Coriobacteriales was increased to a lesser extent, and that of Clostridiales and Bacteroidales was reduced (Fig. 5C).

Bacterial community composition at the family level. At the family level, there were prominent differences between the UC and the control group. The abundance of Enterococcaceae in the UC group was higher than that in the control group. The abundance of Lactobacillaceae and Enterobacteriaceae was also increased in the UC group, and that of Bifidobacteriaceae 
A
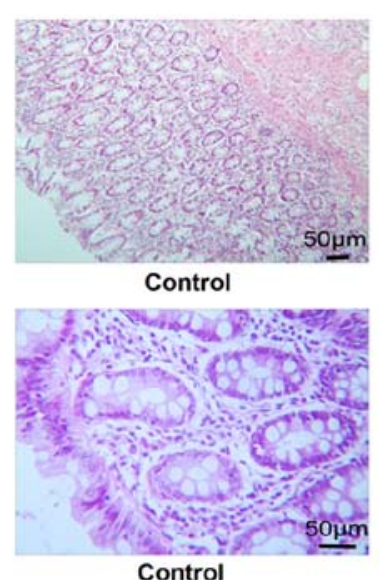

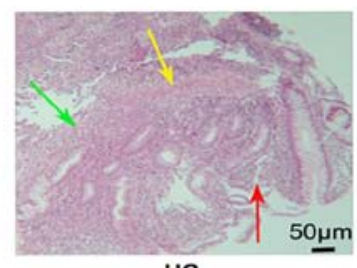

UC

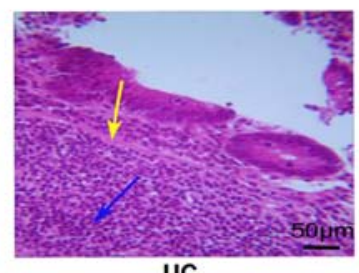

UC
B

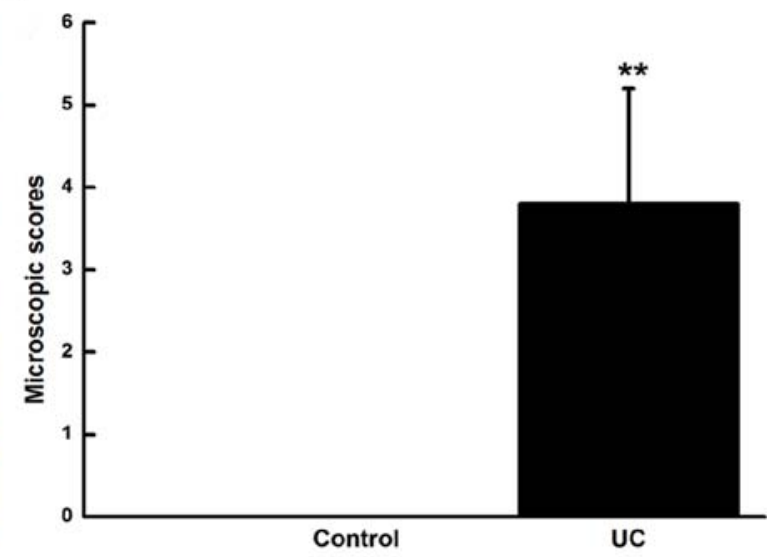

Figure 1. Histopathological evaluation and microscopy scores. (A) Hematoxylin and eosin staining (upper panels, magnification, x100; lower panels, magnification, x200). The arrows reflect fragmentation and loss of the intestinal epithelial layer (red arrow), loss of the crypts of Lieberkühn (green arrow), infiltration of neutrophils and lymphocytes into the mucosal compartment (blue arrow) and manifestation of capillary hyperemia and dilatation (yellow arrow). (B) Histopathological microscopy score. The data are presented as the mean \pm standard deviation. $\mathrm{n}=16$ for UC group, and $\mathrm{n}=10$ for control group. ${ }^{* * *} \mathrm{P}<0.01$ vs. the control group. UC, ulcerative colitis.

A

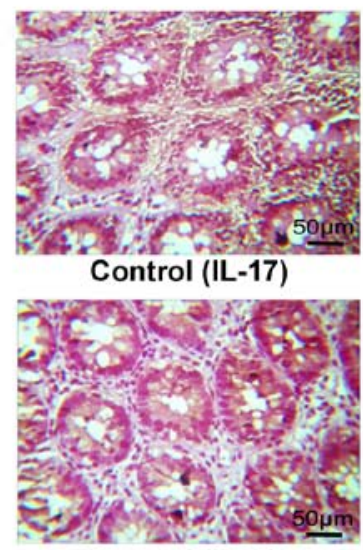

Control (IL-23)

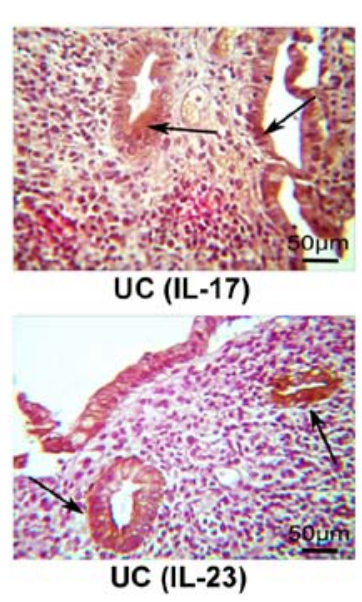

Figure 2. Protein levels of IL-17 and IL-23 in the colon were examined via immunohistochemical staining. (A) Expression of IL-17 and IL-23 in the colon. The arrows highlight the positive staining of IL-17 or IL-23. (B) Quantification of the IL-17 and IL-23 immunohistochemical staining. The data are presented as the mean \pm standard deviation. $\mathrm{n}=16$ for UC group and $\mathrm{n}=10$ for control group. ${ }^{* *} \mathrm{P}<0.01$ vs. the control group. UC, ulcerative colitis; IL-17, interleukin-17; IL-23, interleukin-23.

was slightly increased; however, the abundance of Lachnospiraceae and Bacteroidaceae was decreased (Fig. 5D).

Top five abundant bacteria at the genus level. In the analysis of the species diversity differences between the disease group and the control group by Metastats (http://metastats.cbcb. umd.edu/), the top five species with the greatest abundance differences were Enterococcus $(\mathrm{P}<0.01)$, Lactobacillus $(\mathrm{P}<0.05)$, Escherichia-Shigella $(\mathrm{P}<0.05)$, Bifidobacterium and Bacteroides (Fig. 6). Enterococcus exhibited the highest abundance among the gut bacteria of the disease group.

Key OTU analysis of the gut microbiota. The key OTUs were identified using partial least square discriminate analysis (PLS-DA). The comparison of the control with the UC group revealed that each group had increased numbers of OTUs belonging to different species (data not shown). In the Venn diagram, there were 176 OTUs shared between the two groups.

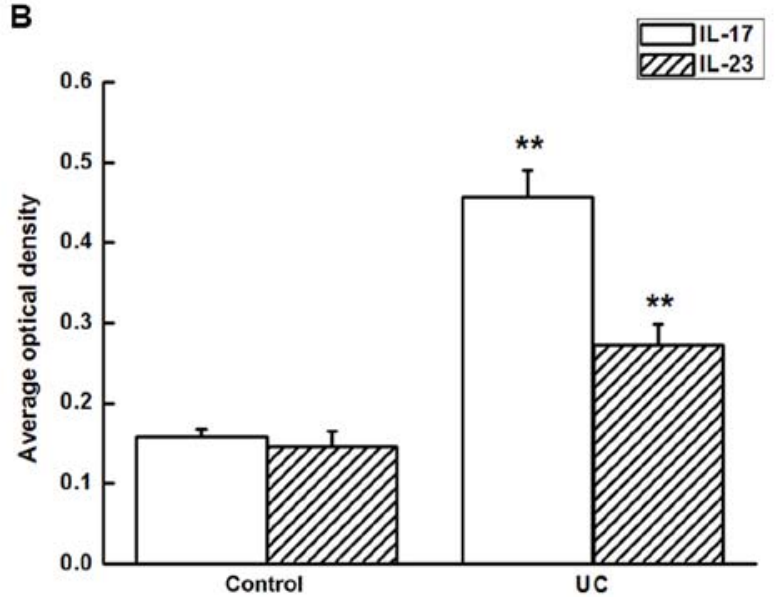

There were 30 types of unique OTUs in the healthy control group and 14 types in the UC group; the two groups displayed a small number of differences in their OTU types (data not shown).

Non-metric multidimensional scaling (NMDS) analysis. The UC and the control group were distributed on both sides of the coordinate axis, they were relatively concentrated in distance, and the stress value was $<0.173$ (Fig. 7). This analysis accurately reflected the differences between the two groups.

Analysis of similarities (ANOSIM). ANOSIM is a non-parametric test, which examines whether the differences between groups ( $\geq 2$ groups) are higher than those within the group, ensuring that the grouping is accurate (Fig. 8). The R-value was obtained via the analysis of the distance between the sample matrices; usually, the R-value range is -1 to 1 , and the current result was between 0 and 1 . An R-value close to zero demonstrates that no significant difference between the groups 

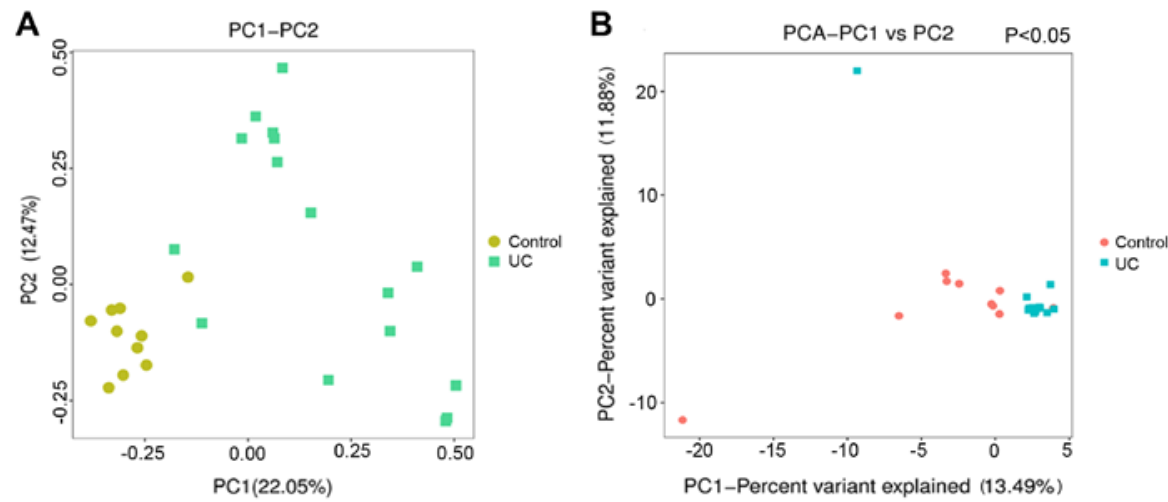

\section{C}

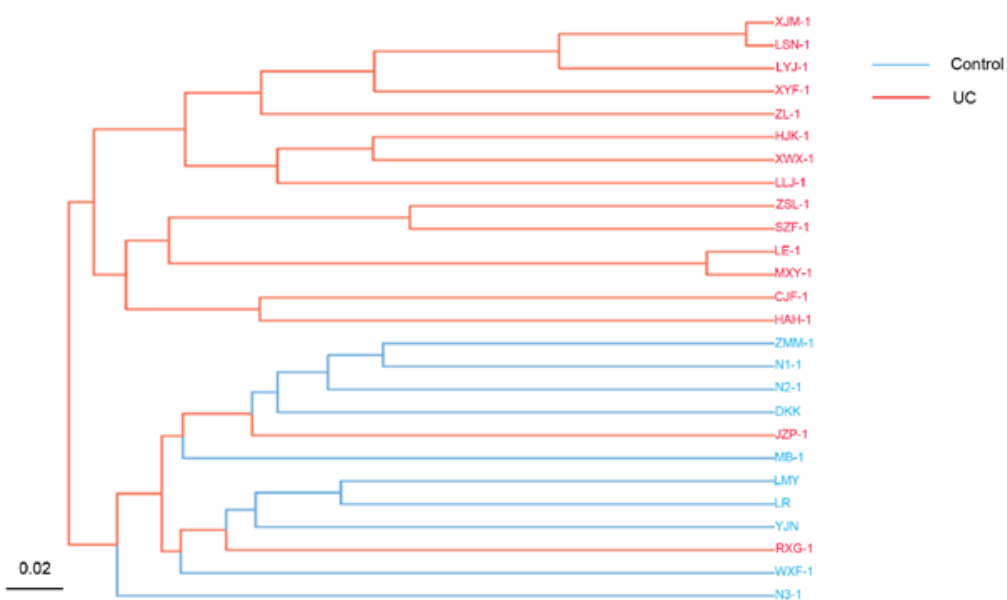

Figure 3. Comparison of the $\beta$-diversity index between patients with UC and individuals without IBD. (A) Principal coordinate analysis and (B) PCA were performed using the $\beta$-diversity metrics based on the weighted UniFrac distance within patients with UC and non-IBD controls. The P-value was calculated using a non-parametric multivariate analysis of variance. There were differences in the composition of the microbial population between patients with UC and non-IBD controls. (C) Analysis of the unweighted pair group method with arithmetic mean clustering between the two groups. UC, ulcerative colitis; IBD, inflammatory bowel disease; PCA, principal component analysis; PC1, principal coordinate 1; PC2, principal coordinate2.

A

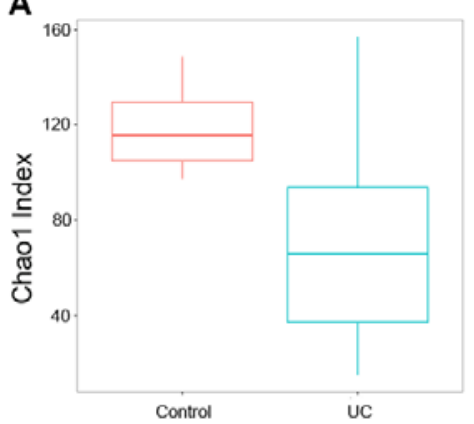

B

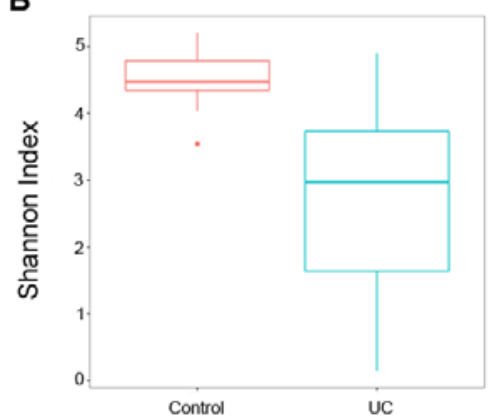

C

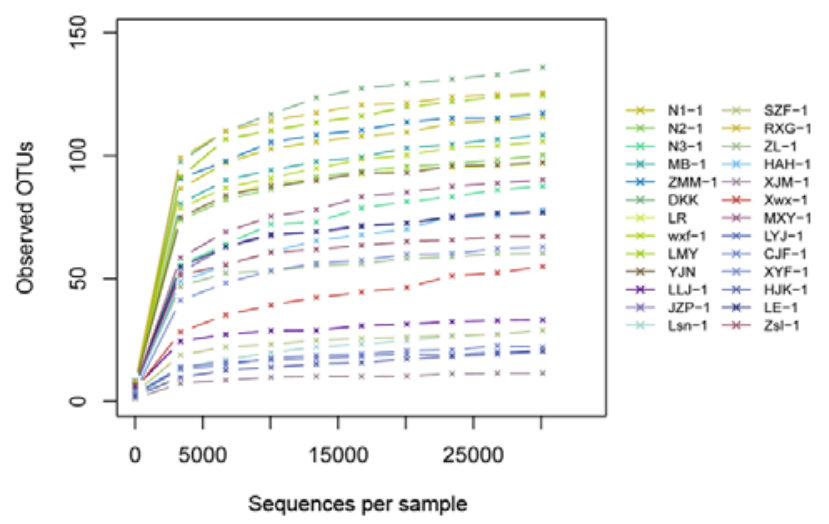

Figure 4. $\alpha$-diversity calculation of biodiversity and rarefaction curves for each sample. (A) Chao1 and (B) Shannon-Wiener index. (C) Rarefaction curves UC, ulcerative colitis; OTUs, operational taxonomic units. 
A

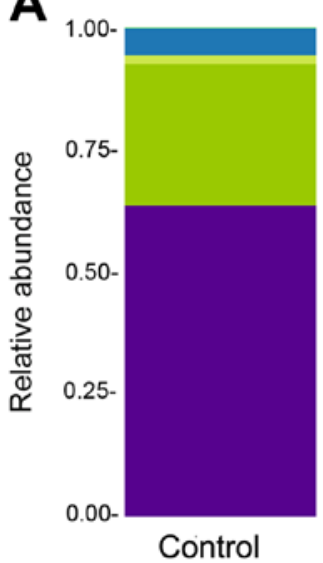

C

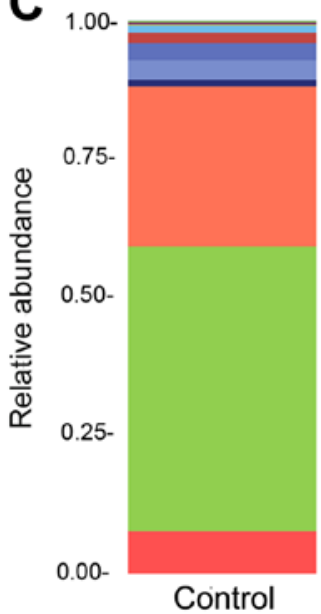

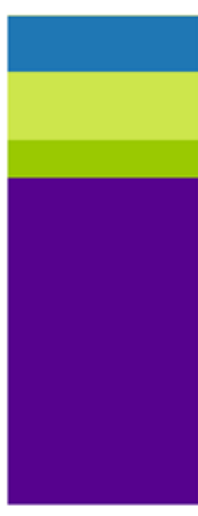

UC

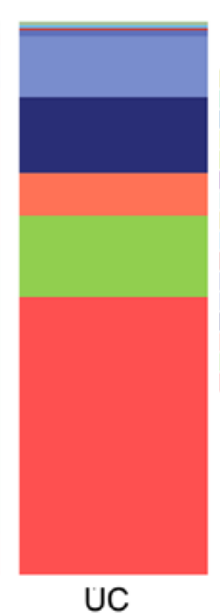

B
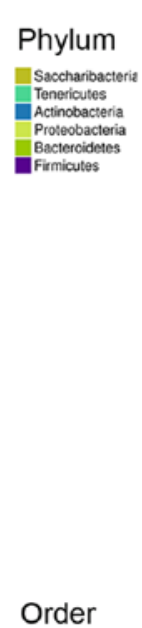

D
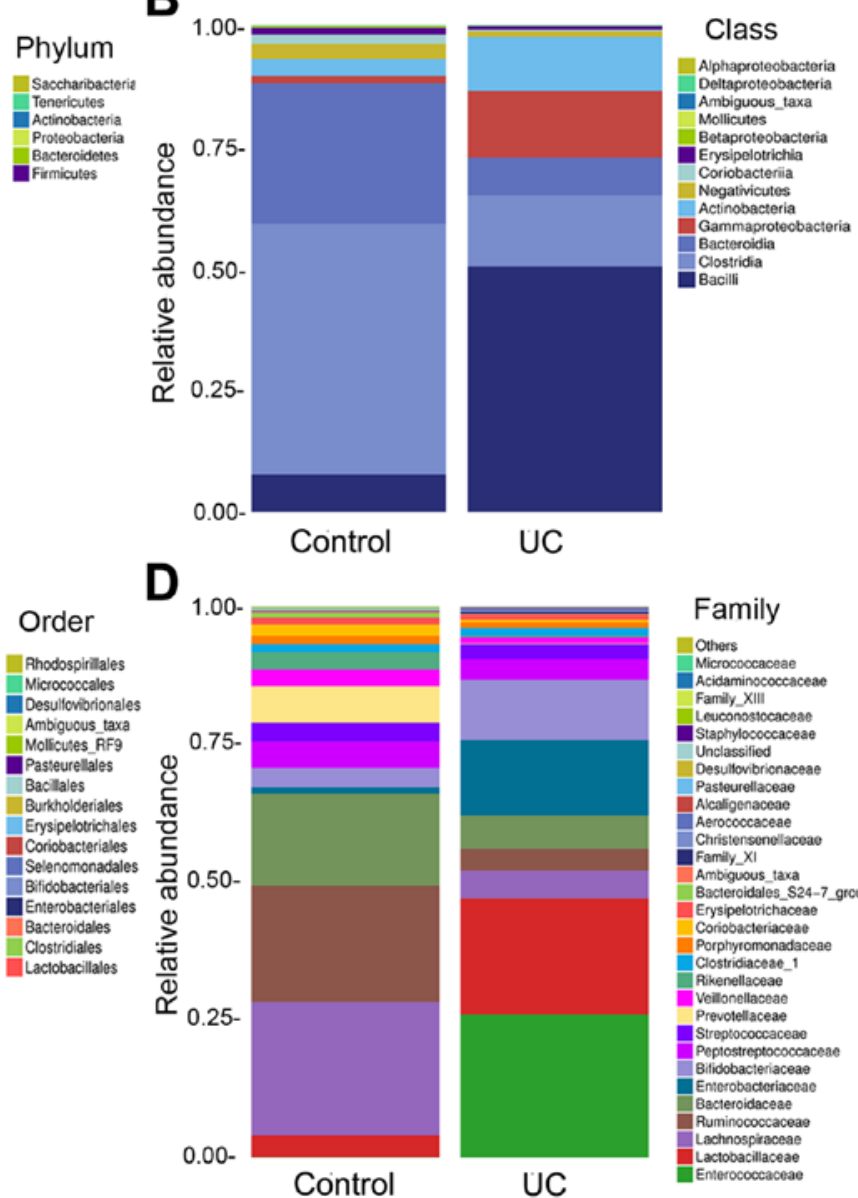
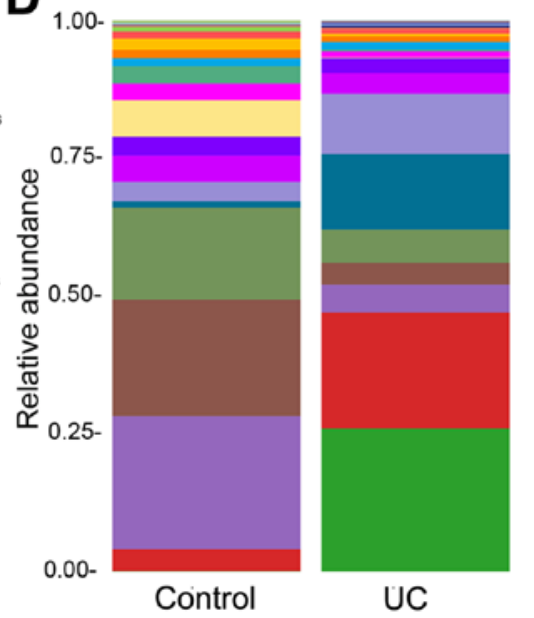

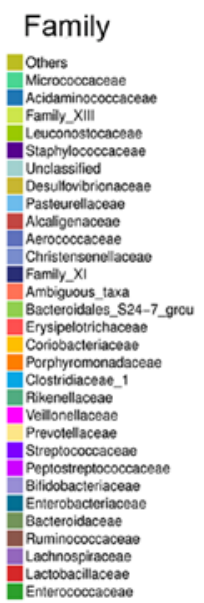

Figure 5. Relative abundances at the phylum, class, order and family level in each sample. (A) The relative abundance of top 30 species at the phylum level. The relative abundance of top 30 species at the (B) class, (C) order and (D) family level. UC, ulcerative colitis.

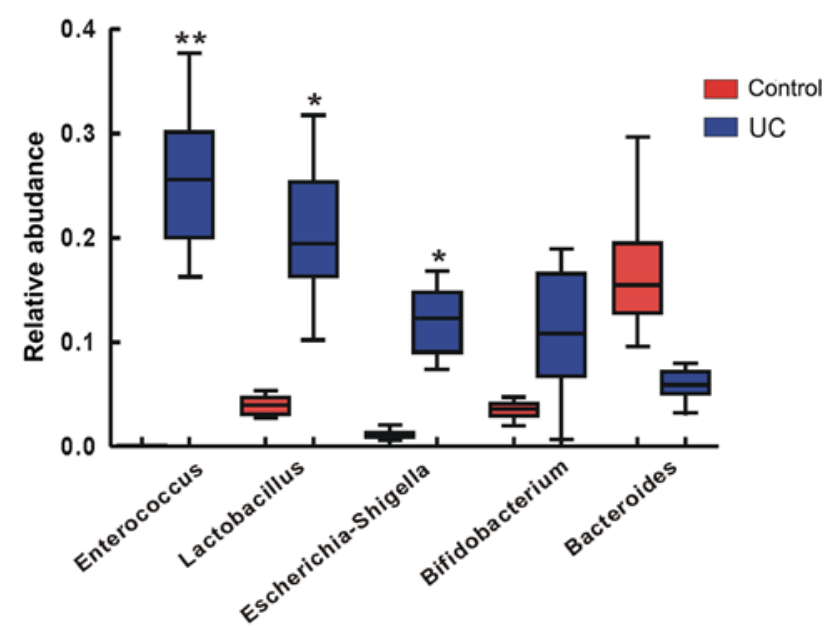

Figure 6. Relative abundances of the top five genera of key operational taxonomic units. MetaStat variation analysis was performed to determine the significance of the species abundance differences between groups. ${ }^{*} \mathrm{P}<0.05$ vs. control; ${ }^{* *} \mathrm{P}<0.01$ vs. control. UC, ulcerative colitis.

exists. The $\mathrm{R}$ value was close to 1 in the present comparison, indicating that the between-group difference was higher than the within-group difference. The P-value represents the reliability of the statistical analysis, and $\mathrm{P}<0.05$ indicated a statistical significance. In the current two groups of samples, the R-value was 0.602, and the P-value was 0.001, which indicated that a significant difference between the two groups was observed, and the analysis was credible.

Correlation between the expression of $I L-17 / I L-23$ and the histopathological score. The average optical density values of IL-17 $(\rho=0.669 ; P=0.035$; Table II) and IL-23 $(\rho=0.733$; $\mathrm{P}=0.016$; Table II) were positively correlated with the histological score.

Correlation between the expression of IL-17/IL-23 and intestinal bacteria. Pearson's correlation analysis revealed that the average optical density of IL-17 was positively correlated with the abundance of Enterococcus $(\mathrm{r}=0.843 ; \mathrm{P}<0.001)$, Lactobacillus $(\mathrm{r}=0.737 ; \mathrm{P}=0.001)$, Bifidobacterium $(\mathrm{r}=0.773$; $\mathrm{P}<0.001)$ and Escherichia-Shigella $(\mathrm{r}=0.663 ; \mathrm{P}=0.005)$. The average optical density of IL-23 was positively correlated with the abundance of Enterococcus $(\mathrm{r}=0.771 ; \mathrm{P}<0.001)$, Lactobacillus $(\mathrm{r}=0.566 ; \mathrm{P}=0.022)$, Bifidobacterium $(\mathrm{r}=0.517$; $\mathrm{P}=0.041)$ and Escherichia-Shigella $(\mathrm{r}=0.613 ; \mathrm{P}=0.012)$. The results are presented in Table III.

\section{Discussion}

The human body is estimated to be composed of $3 \times 10^{13}$ eukaryotic cells and $3.9 \times 10^{13}$ colonizing microorganisms, 
Table II. Correlation of IL-17/IL-23 with the histological score.

\begin{tabular}{lcc}
\hline Inflammatory cytokines & $\rho$ & P-value \\
\hline IL-17 & 0.669 & 0.035 \\
IL-23 & 0.733 & 0.019 \\
\hline
\end{tabular}

IL, interleukin.

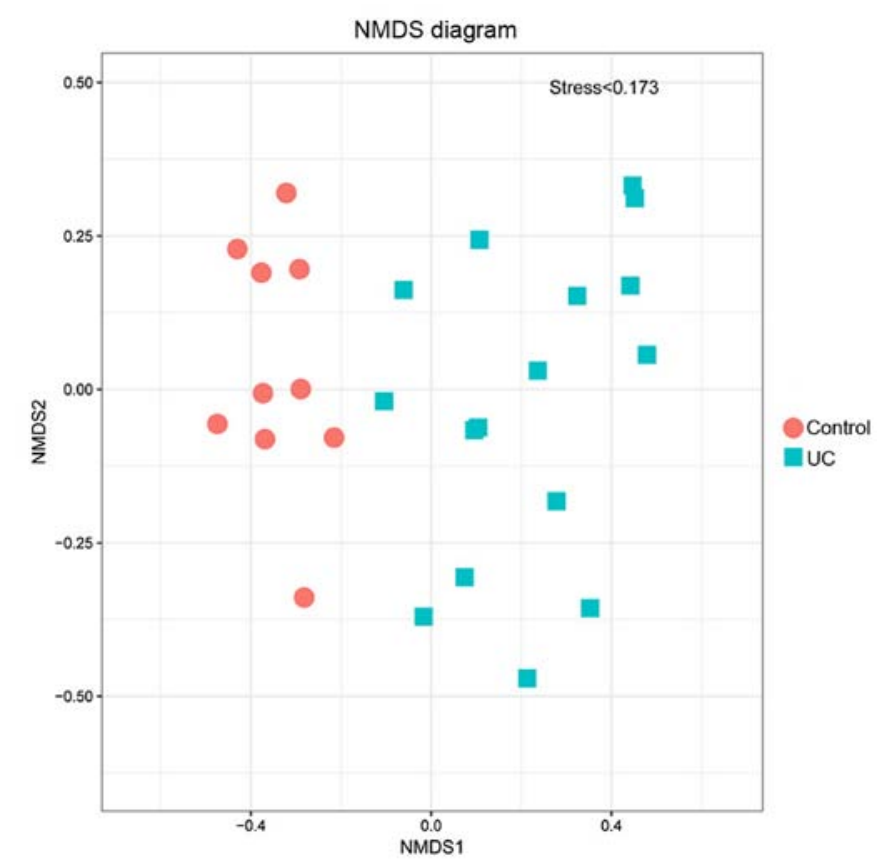

Figure 7. NMDS analysis. The stress value $<0.173$, which reflects the significance of the difference among the samples. UC, ulcerative colitis; NMDS, non-metric multidimensional scaling.

indicating that host cells and microbiota are represented in similar numbers within an individual (25). The gut microbiota constitutes a complex intestinal microecosystem and serves an important role in the host intestinal immune system, the synthesis and absorption of nutrients and the inhibition of colonization by pathogenic bacteria $(26,27)$. An alteration in the bacterial numbers and proportions results in an imbalance in the intestinal mucosa, as well as in the dysfunction of the intestinal epithelial cells and the dysregulation of the immune response $(28,29)$.

Several lines of evidence support the hypothesis that gut microbiota dysbiosis contributes to the pathogenesis of $\operatorname{IBD}(30,31)$. Certain gene knockout or gene-deficient mice did not develop UC when they were placed in a sterile environment, but developed UC following an intestinal flora enema (32), which introduced the concept of 'sterility without inflammation'. It has also been suggested that the composition of the intestinal flora is an important factor that is associated with the pathogenesis of enteritis. Gradel et al (33) demonstrated that patients infected with Campylobacter or Salmonella were susceptible to IBD. Li et al (34) observed fecal bacteria in 16 healthy controls and 41 patients with UC, and revealed that the microbial diversity of the healthy control feces was higher than that of patients with UC. Compared with that in

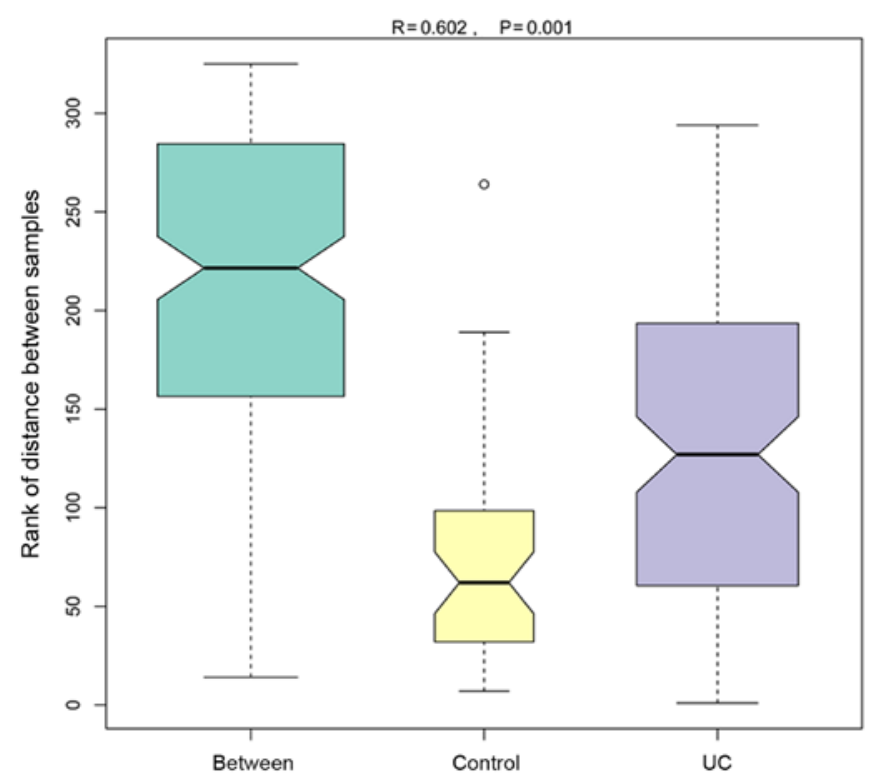

Figure 8. Analysis of similarities. This is a non-parametric test to determine whether the differences between groups ( $\geq 2$ groups) are higher than those within each group. For the two groups of samples, the R-value was 0.602 , and the P-value was 0.001 , which indicated that there was a statistically significant difference between the two groups. UC, ulcerative colitis.

the normal, static and mild active stages, the abundance of the principal types of fecal bacteria were prominently altered in the moderate and severe active stages, and the proportion of the principal bacteria types was negatively correlated with disease activity. Additionally, the number of Clostridium perfringens was increased, and the number of Enterobacteriaceae and rectal bacteria was decreased in patients with UC. The results from Rajilić-Stojanović et al (35) revealed that the composition of fecal bacteria in patients with UC was different from that in healthy controls. In the active disease period, the primary markers of bacterial dysbiosis were as follows: The bacterial diversity of the Clostridium IV cluster was decreased, the abundance of bacteria involved in the metabolism of butyric and propionic acid, including Rumen, Enterococcus, Roche and Akkermansia, was decreased, however, the abundance of the conditionally pathogenic bacteria Clostridium, Streptococcus, Helicobacter, Campylobacter and Clostridium difficile was increased. Other studies have also indicated that microbiota alterations, namely dysbiosis, are common in IBD (36-39). These alterations include an increased number of Enterobacteriaceae and Bacteroides-Prevotella, an increased or decreased abundance of Bifidobacteria and a decrease in the numbers of Firmicutes (especially Clostridium coccoides, Eubacterium rectale and Faecalibacterium prausnitzii). Therefore, recent technological advances have provided evidence that gut dysbiosis is one of the triggers and/or mediators of the progression of intestinal inflammation in IBD.

In the current study, a prominent decrease in the abundance of Bacteroides in the UC group was observed, accompanied by an increased abundance of Proteus and Actinomycetes, as well as a slightly increased abundance of Firmicutes. These results are not consistent with the results of other reports (40-42). Several studies (43-46) have indicated that the reduced diversity of the gut microbiota in patients with IBD is primarily associated with the reduced abundance of the phylum Firmicutes, 
Table III. Correlation of IL-17/IL-23 with top five differentially abundant bacteria.

\begin{tabular}{lcccc}
\hline Bacteria & r (IL-17) & P-value (IL-17) & r (IL-23) & P-value (IL-23) \\
\hline Enterococcus & 0.843 & $<0.001$ & 0.771 & $<0.001$ \\
Lactobacillus & 0.737 & 0.001 & 0.566 & 0.022 \\
Bacteroides & 0.454 & 0.077 & 0.475 & 0.063 \\
Bifidobacterium & 0.773 & $<0.001$ & 0.517 & 0.041 \\
Escherichia-Shigella & 0.663 & 0.005 & 0.613 & 0.012 \\
\hline
\end{tabular}

IL, interleukin.

particularly the Clostridium cluster IV of anaerobic bacteria. The reason for this difference may be accounted for by the samples being collected from different research centers, therefore additional supporting evidence is required.

The increased appreciation of the role of the microbiota in host physiology and disease has resulted in a vigorous effort to understand the precise mechanisms underlying the involvement of the microbiota in the pathogenesis of IBD (47). The alterations in bacterial numbers and proportions result in an ecological imbalance in the intestinal mucosa, a decreased production of metabolites, butyrate and anti-inflammatory factors, and an increased production of pro-inflammatory factors (48). This is followed by a dysfunction of intestinal epithelial cell responses to the gut flora signals, and a dysregulation of the signal transfer and the immune responses (49). Dysbacteriosis also leads to a lack of necessary micronutrients, such as short chain fatty acids, and redox potential in the intestinal mucosa, thereby resulting in increased permeability and damage of the intestinal mucosa $(28,29)$.

Another important manifestation of dysbacteriosis is the alteration in the microbiota composition. Several studies have indicated that the bacterial diversity was reduced in patients with UC (50-52), however, no differences were observed in the gut microbiota diversity between patients with CD and those with UC, but prominent differences existed between the microbiota of patients with IBD and healthy controls. In addition, $\alpha$-diversity was demonstrated to be lower in patients with UC and CD compared with non-IBD controls (53). In the present study, microbial $\alpha$-diversity was substantially decreased in the UC compared with the healthy control group, as assessed by the Chaol and Shannon indexes, suggesting that the composition of the microbiome may be different between the two groups. The hypothesis of decreased $\alpha$-diversity contends that various intestinal bacteria constitute a normal barrier (54). In response to various stresses, such as inflammation and antibiotics, the microbial diversity decreases, the normal bacterial functions cannot be performed and anecological imbalance occurs, which indicates that the gut microbiota diversity is required to maintain balance $(13,46,55,56)$.

The $\beta$-diversity, which reflects the diversity of constituents in different samples, was also dissimilar between the two groups. PCoA is a method of research data visualization to analyze similarities or differences, and involves ranking the samples through a series of eigenvalues and eigenvectors, and subsequently selecting the most prominent of several main characteristic values to calculate the coordinates of the main distance matrix (57). In the present study, the principal coordinate sample difference contribution rate was $22.05 \%$, the second was $12.47 \%$, and the two groups were distinctly separated based on the distinguished coordinates. Similarly, the PCA and NMDS results also supported this conclusion. In patients with IBD, differences in the abundances of pro-inflammatory and non-inflammatory microbiota have been identified based on $\beta$-diversity (48). The exploration of inflamed and non-inflamed sites per patient also resulted in similar conclusions (58).

At the class level, the Bacillus class was the most abundant in the UC group, and the abundance of Gammaproteobacteria also appeared to increase, compared with that in the control group. By contrast, the abundance of Clostridia and Bacteroidia was reduced. Bacilli, as a taxonomic class of bacteria, include two orders, Bacillales and Lactobacillales. Lactobacilli have been identified as probiotics that can alter the intestinal environment and cure or alleviate certain intestinal diseases (59). Lactobacillus bulgaricus OLL1181 has been reported to activate the aryl hydrocarbon receptor pathway to suppress inflammation (60). Animal experiments have indicated that Lactobacillus plantarum K68 improved the dextran sulfate sodium-induced UC in BALB/c mice via anti-inflammatory and immunomodulatory activities (61). Furthermore, Lactobacillales BR11 were demonstrated to reduce the severity of experimental IBD due to their probiotic properties, possibly via the production of thiol using a unique cysteine/cystine-transport system (62). Contrary to these previous studies, in the current study, the UC group had the highest levels of Lactobacillus, and at the genus level, Lactobacillus also displayed high abundance. It was hypothesized that $\beta$-diversity differences existed between the UC group and healthy controls, but this was not observed in certain species of bacteria, such as Lactobacillus and Bifidobacterium $(63,64)$. Therefore, the clinical function of probiotics for the treatment of intestinal diseases was questioned. Indeed, the therapeutic effects of some probiotics were not satisfactory (65). In a double-blind, randomized, placebo-controlled study, VSL\# ${ }^{\circledR}$, which is a high-potency probiotic mixture that has been efficiently used in the treatment of pouchitis, was used to treat relapsing patients with UC, who were also being treated with 5-aminosalicylic acid and/or immunosuppressants at stable doses (27). Although VSL\#3 appeared to exert an alleviative effect, no significant differences were observed in the measured parameters. The association of Lactobacillus with UC should be the focus of future research. 
Although the gut microbiota has important physiological functions, it may also pose a considerable threat to the immune homeostasis of the host. Bacteria may induce an immune response if they are recognized by mucosal immune cells as foreign species (66). In the present study, it was also demonstrated that Enterococcus was the most abundant genus in the UC group, which was clearly distinct from the control group. Furthermore, in the bacterial abundance diagram, the difference in the abundance of Enterococcus was higher compared with other bacteria. These results are consistent with those of previous studies, which aimed at elucidating the association between Enterococcus faecalis and chronic intestinal inflammation. Steck et al (67) reported that Enterococcus resulted in chronic intestinal inflammation via producing gelatinase GelE, which is a type of metalloproteinase that can damage the intestinal mucosal barrier. Another study also demonstrated that Enterococcus faecalis was enriched in patients with IBD, and an increased Enterococcus faecalis abundance was associated with clinically active CD (68). However, the mechanisms underlying the pathogenesis of Enterococcus remain to be further elucidated.

Previous studies investigating IL-17 and IL-23 demonstrated that these two factors were associated with intestinal inflammation. Xie et al (69) revealed that the occurrence of human colon cancer was associated with an increased inflammatory response driven by IL-17. Another study, which investigated the lamina propria $T$ cells of the intestinal mucosa, revealed that an increased IL-17 expression and a reduced suppressor activity were observed in patients with UC compared with the control group (70). In a meta-analysis, the level of IL-17 was indicated to increase in association with the increase of the degree of UC (17). Furthermore, drugs inhibiting the IL-17/IL-23 axis were demonstrated to exhibit a therapeutic effect on UC (18,71). In summary, IL-17 and IL-23 were associated with intestinal inflammation. Previous studies have comprehensively described the intestinal flora of patients with IBD, suggested that the alterations of the IBD microbiome are closely associated with the development of inflammation, identified the function of certain cells and mechanisms which regulate the interaction between the host and the microorganisms, or explored the flora of patients with UC with/without primary biliary cirrhosis (46,72-76). In the current study, the altered intestinal flora and IL-17/IL-23 expression were discussed, and it was demonstrated that IL-17 and IL-23 expression was correlated with histological score. The association of IL-17/IL-23 with intestinal flora was also analyzed, and it was revealed that IL-17/IL-23 expression was correlated with the abundance of Enterococcus, Lactobacillus, Bifidobacterium and Escherichia-Shigella.

However, certain limitations are still present in this field. For example, the challenges of the microbiota studies, which are associated with the remarkable variation in the microbiome, owing to a variety of factors, including body site, age, location, lifestyle, diet and host genetics, still exist, and the importance of isolation standards and sample processing in these studies requires improvement $(75,77)$. In addition, as demonstrated in early genetic association studies $(75,77,78)$, the current studies investigating the microbiota may not be adequate to identify the microbiota-phenotype associations, and future studies are likely to require an increased numbers of samples. Whether a more general intestinal microbial profile of fecal samples or a specific localized profile of endoscopic tissue samples is optimal for the study of microbiota in UC remains to be elucidated. The mucosa-associated organisms, however, are not limited to a particular intestinal location and are observed in fecal and tissue samples, albeit at different abundances (14). Until a consensus is reached on the optimal biospecimen type for the characterization of the intestinal microbiota, future studies may consider collecting both biospecimen types in their design. Next generation sequencing technologies continue to develop, with their cost progressively decreasing, which results in an increase in the number of sequences that are generated per sample (78). As a consequence, more in-depth sequencing will be feasible in future studies, resulting in low-abundance organisms being characterized.

Despite the results of the present study, it is possible that the presence of the pathogens examined in fecal samples may not accurately reflect the microbiome dynamics in the gut. Adherent bacteria are in direct contact with the host epithelium, and may exert greater effects on gene expression in the colonic mucosal cells than transient bacteria, which are removed in fecal samples (79). Therefore, the lack of mucosal microbiota is a potential limitation of the current study. As the bacterial abundance in the colonic tissue is low, and the small size of the collected tissues was not adequate for the detection of microbiota, fecal samples were selected, which reflect the colonization of flora to a certain extent. In addition, the number of patients in the current study was small. However, more patients will be included in order to collect adequate samples and deeply sequence each sample.

In conclusion, the present study indicated that the intestinal microbiota of patients with UC was different from that of healthy controls at multiple taxonomic levels, and an altered intestinal microflora was closely associated with the degree of inflammation. The IL-23/IL-17 axis, as a key factor in the development of UC, maybe associated with the alterations of intestinal microflora. The interaction between intestinal microflora and the IL-23/IL-17 axis may serve an important role in the pathogenesis of UC.

\section{Acknowledgements}

Not applicable.

\section{Funding}

The present study was funded by the National Natural Science Foundation of China (grant nos. 81500430 and U1304802 to Dr Xu Hong Lin), Henan Medical Science and Technology Tackling Project (grant no. 201702136 to Mr. Zhi Feng Dai; grant no. 2018020320 to Mr. Hui Chao Wang), the Key Project of Science and Technology Research of the Education Department of Henan (grant no. 17A320019 to Mr. Hui Chao Wang), Research of the Shanghai Municipal Commission of Health and Family Planning (grant no. 201440313 to Dr Kun Li) and the Science and Technology Planning Project of Henan Province (grant nos. 192102310045 and 182102310544 to Dr Xu Hong Lin; grant no. 182102310566 to Mr. Hui Chao Wang; grant no. 182102310567to Mrs. Rui Lin Yang; grant no. 172102310150 to $\mathrm{Mr}$. Yao Xu). 


\section{Availability of data and materials}

The datasets used and/or analyzed during the current study are available from the corresponding author on reasonable request.

\section{Authors' contributions}

XHL designed the research protocols and revised the manuscript. ZFD, XYM and RLY performed the experiments and wrote the manuscript. HCW, DDX, XBG, SSM, RX and JNY acquired and analyzed the data and performed the experiments. YXL, YX and KL helped to prepare the figures and tables. DDX, XBG, YXL, KL and YX interpretated and confirm the authenticity of all the raw data. All authors have read and approved the final manuscript.

\section{Ethics approval and consent to participate}

Written informed consent was obtained from each patient prior to the procedure. Ethical approval was obtained from the medical Ethics Committee of Huaihe Hospital of Henan University (Kaifeng, China).

\section{Patient consent for publication}

Not applicable.

\section{Competing interests}

The authors declare that they have no competing interests.

\section{References}

1. Sheehan D, Moran C and Shanahan F: The microbiota in inflammatory bowel disease. J Gastroenterol 50: 495-507, 2015.

2. Sun M, Wu W, Liu Z and Cong Y: Microbiota metabolite short chain fatty acids, GPCR, and inflammatory bowel diseases. J Gastroenterol 52: 1-8, 2017.

3. Rogler G: Chronic ulcerative colitis and colorectal cancer. Cancer Lett 345: 235-241, 2014.

4. Adams SM and Bornemann PH: Ulcerative colitis. Am Fam Physician 87: 699-705, 2013.

5. Al Bander Z, Nitert MD, Mousa A and Naderpoor N: The gut microbiota and inflammation: An overview. Int J Environ Res Public Health 17: 7618, 2020.

6. Yap YA and Mariño E: An insight into the intestinal web of mucosal immunity, microbiota, and diet in inflammation. Front Immunol 9: 2617, 2018.

7. Shen ZH, Zhu CX, Quan YS, Yang ZY, Wu S, Luo WW, Tan B and Wang XY: Relationship between intestinal microbiota and ulcerative colitis: Mechanisms and clinical application of probiotics and fecal microbiota transplantation. World J Gastroenterol 24: 5-14, 2018.

8. Jostins L, Ripke S, Weersma RK, Duerr RH, McGovern DP, Hui KY, Lee JC, Schumm LP, Sharma Y, Anderson CA, et al: Host-microbe interactions have shaped the genetic architecture of inflammatory bowel disease. Nature 491: 119-124, 2012.

9. Macdonald TT and Monteleone G: Immunity, inflammation, and allergy in the gut. Science 307: 1920-1925, 2005.

10. Kostic AD, Xavier RJ and Gevers D: The microbiome in inflammatory bowel disease: Current status and the future ahead. Gastroenterology 146: 1489-1499, 2014.

11. Nishikawa J, Kudo T, Sakata S, Benno Y and Sugiyama T: Diversity of mucosa-associated microbiota in active and inactive ulcerative colitis. Scand J Gastroentero 144: 180-186, 2009.

12. Ma XY, Dai ZF, Wang HC, Yang JN, Tang XY, Kang YH, Ding CS, Li YX, Yang RL and Lin XH: Change of intestinal flora and its relationship with IL-23 /IL-17 axis in patients with ulcerative colitis Chinese Journal of Pathophysiology 34: 884-892, 2008 (In Chinese).
13. Sartor RB and Wu GD: Roles for intestinal bacteria, viruses, and fungi in pathogenesis of inflammatory bowel diseases and therapeutic approaches. Gastroenterology 152: 327-339.e4, 2017.

14. Gevers D, Kugathasan S, Denson LA, Vázquez-Baeza Y, Van Treuren W, Ren B, Schwager E, Knights D, Song SJ, Yassour M, et al: The treatment-naive microbiome in new-onset Crohn's disease. Cell Host Microbe 15: 382-392, 2014.

15. Sokol H and Seksik P: The intestinal microbiota in inflammatory bowel diseases: Time to connect with the host. Curr Opin Gastroenterol 26: 327-331, 2010.

16. Yu P, Shen F, Zhang X, Cao R, Zhao X, Liu P, Tu H, Yang X, Shi R and Zhang $\mathrm{H}$ : Association of single nucleotide polymorphisms of IL23R and IL17 with ulcerative colitis risk in a Chinese Han population. PLoS One 7: e44380, 2012.

17. Li J, Tian H, Jiang HJ and Han B: Interleukin-17 SNPs and serum levels increase ulcerative colitis risk: A meta-analysis. World J Gastroenterol 20: 15899-15909, 2014.

18. Larussa T, Oliverio M, Suraci E, Greco M, Placida R, Gervasi S, Marasco R, Imeneo M, Paolino D, Tucci L, et al: Oleuropein decreases cyclooxygenase- 2 and interleukin-17 expression and attenuates inflammatory damage in colonic samples from ulcerative colitis patients. Nutrients 9: 391, 2017.

19. Inflammatory bowel disease collaboration group of Chinese Society of Gastroenterology: Consensus on the diagnosis and treatment of inflammatory bowel disease in China. Chin J Intern Med 47: 73-79, 2008 (In Chinese).

20. Liang J,Zhou L, Sha SM, Lei SN, Luo GH and Wu KC: Consensus on Diagnosis and Management of Inflammatory Bowel Disease (Guangzhou, 2012), an interpretation on the diagnosis of UC. Chin J Gastroenterol 17: 712-720, 2012 (In Chinese).

21. Siegmund B, Lehr HA, Fantuzzi G and Dinarello CA: IL-1 $\beta$-converting enzyme (caspase-1) in intestinal inflammation. Proc Natl Acad Sci USA 98: 13249-13254, 2001.

22. Kim BR, Shin J, Guevarra R, Lee JH, Kim DW, Seol KH, Lee JH, Kim HB and Isaacson R: Deciphering diversity indices for a better understanding of microbial communities. J Microbiol Biotechnol 27: 2089-2093, 2017.

23. Ren G, Yu M, Li K, Hu Y, Wang Y, Xu X and Qu J: Seleno-lentinan prevents chronic pancreatitis development and modulates gut microbiota in mice. J Funct Foods 22: 177-188, 2016.

24. Schmidt M, Unterer S, Suchodolski JS, Honneffer JB, Guard BC, Lidbury JA, Steiner JM, Fritz J and Kölle P: The fecal microbiome and metabolome differs between dogs fed bones and raw food (BARF) diets and dogs fed commercial diets. PLoS One 13: e201279, 2018.

25. Sender R, Fuchs S and Milo R: Are we really vastly outnumbered? Revisiting the ratio of bacterial to host cells in humans. Cell 164: 337-340, 2016.

26. Tomasello G, Bellavia M, Palumbo VD, Gioviale MC, Damiani P and Lo Monte AI: From gut microflora imbalance to mycobacteria infection: Is there a relationship with chronic intestinal inflammatory diseases? Ann Ital Chir 82: 361-368, 2011.

27. Shen J, Zuo ZX and Mao AP: Effect of probiotics on inducing remission and maintaining therapy in ulcerative colitis, Crohn's disease, and pouchitis: Meta-analysis of randomized controlled trials. Inflamm Bowel Dis 20: 21-35, 2014.

28. Verma R, Verma AK, Ahuja V and Paul J: Real-time analysis of mucosal flora in patients with inflammatory bowel disease in India. J Clin Microbiol 48: 4279-4282, 2010.

29. Marteau P: Bacterial flora in inflammatory bowel disease. Dig Dis 27 (Suppl 1): S99-S103, 2009.

30. Mosca A, Leclerc M and Hugot JP: Gut microbiota diversity and human diseases: Should we reintroduce key predators in our ecosystem? Front Microbiol 7: 455, 2016.

31. Manichanh C, Rigottier-Gois L, Bonnaud E, Gloux K, Pelletier E, Frangeul L, Nalin R, Jarrin C, Chardon P, Marteau P, et al: Reduced diversity of faecal microbiota in Crohn's disease revealed by a metagenomic approach. Gut 55: 205-211, 2006.

32. Balish E and Warner T: Enterococcus faecalis induces inflammatory bowel disease in interleukin-10 knockout mice. Am J Pathol 160: 2253-2257, 2002.

33. Gradel KO, Nielsen HL, Schonheyder HC, Ejlertsen T, Kristensen B and Nielsen H: Increased short- and long-term risk of inflammatory bowel disease after salmonella or campylobacter gastroenteritis. Gastroenterology 137: 495-501, 2009.

34. Li KY, Wang JL, Wei JP, Gao SY, Zhang YY, Wang LT and Liu G: Fecal microbiota in pouchitis and ulcerative colitis. World J Gastroenterol 22: 8929-8939, 2016. 
35. Rajilić-Stojanović M, Shanahan F, Guarner F and de Vos WM: Phylogenetic analysis of dysbiosis in ulcerative colitis during remission. Inflamm Bowel Dis 19: 481-488, 2013.

36. Cucchiara S, Iebba V, Conte MP and Schippa S: The microbiota in inflammatory bowel disease in different age groups. Dig Dis 27: 252-258, 2009.

37. Swidsinski A, Loening-Baucke V, Vaneechoutte $M$ and Doerffel Y: Active Crohn's disease and ulcerative colitis can be specifically diagnosed and monitored based on the biostructure of the fecal flora. Inflamm Bowel Dis 14: 147-161, 2008.

38. Sokol H, Seksik P, Furet JP, Firmesse O, Nion-Larmurier I, Beaugerie L, Cosnes J, Corthier G, Marteau P and Doré J: Low counts of Faecalibacterium prausnitzii in colitis microbiota. Inflamm Bowel Dis 15: 1183-1189, 2009.

39. Schwiertz A, Jacobi M, Frick JS, Richter M, Rusch K and Köhler H: Microbiota in pediatric inflammatory bowel disease. J Pediatr 157: 240-244.e1, 2010.

40. Kabeerdoss J, Jayakanthan P, Pugazhendhi S and Ramakrishna BS Alterations of mucosal microbiota in the colon of patients with inflammatory bowel disease revealed by real time polymerase chain reaction amplification of $16 \mathrm{~S}$ ribosomal ribonucleic acid. Indian J Med Res 142: 23-32, 2015.

41. Dziarski R, Park SY, Kashyap DR, Dowd SE and Gupta D: Pglyrp-regulated gut microflora prevotella falsenii, parabacteroides distasonis and Bacteroides eggerthii enhance and alistipes finegoldii attenuates colitis in mice. PLoS One 11: e146162, 2016

42. Swidsinski A, Weber J, Loening-Baucke V, Hale LP and Lochs H: Spatial organization and composition of the mucosal flora in patients with inflammatory bowel disease. J Clin Microbiol 43 3380-3389, 2005.

43. Bamola VD, Ghosh A, Kapardar RK, Lal B, Cheema S, Sarma P and Chaudhry R: Gut microbial diversity in health and disease: Experience of healthy Indian subjects, and colon carcinoma and inflammatory bowel disease patients. Microb Ecol Health Dis 28: 1322447, 2017.

44. Frank DN, St Amand AL, Feldman RA, Boedeker EC, Harpaz N and Pace NR: Molecular-phylogenetic characterization of microbial community imbalances in human inflammatory bowel diseases. Proc Natl Acad Sci USA 104: 13780-13785, 2007.

45. Dong LN, Wang M, Guo J and Wang JP: Role of intestinal microbiota and metabolites in inflammatory bowel disease. Chin Med J (Engl) 132: 1610-1614, 2019.

46. Becker C, Neurath MF and Wirtz S: The intestinal microbiota in inflammatory Bowel Disease. ILAR J 56: 192-204, 2015.

47. Nagao-Kitamoto $\mathrm{H}$ and Kamada N: Host-microbial cross-talk in inflammatory bowel disease. Immune Netw 17: 1-12, 2017.

48. Neurath MF: Cytokines in inflammatory bowel disease. Nat Rev Immunol 14: 329-342, 2014.

49. McDermott AJ and Huffnagle GB: The microbiome and regulation of mucosal immunity. Immunology 142: 24-31, 2014.

50. Ott SJ, Musfeldt M, Wenderoth DF, Hampe J, Brant O, Fölsch UR, Timmis KN and Schreiber S: Reduction in diversity of the colonic mucosa associated bacterial microflora in patients with active inflammatory bowel disease. Gut 53: 685-693, 2004.

51. Kabeerdoss J, Sankaran V, Pugazhendhi S and Ramakrishna BS: Clostridium leptum group bacteria abundance and diversity in the fecal microbiota of patients with inflammatory bowel disease: A case-control study in India. BMC Gastroenterol 13: 20, 2013.

52. Imhann F, Vich Vila A, Bonder MJ, Fu J, Gevers D, Visschedijk MC, Spekhorst LM, Alberts R, Franke L, van Dullemen HM, et al: Interplay of host genetics and gut microbiota underlying the onset and clinical presentation of inflammatory bowel disease. Gut 67: 108-119, 2018.

53. Nishino K, Nishida A, Inoue R, Kawada Y, Ohno M, Sakai S Inatomi O, Bamba S, Sugimoto M, Kawahara M, et al: Analysis of endoscopic brush samples identified mucosa-associated dysbiosis in inflammatory bowel disease. J Gastroenterol 53: 95-106, 2018.

54. Danilova NA, Abdulkhakov SR, Grigoryeva TV, Markelova MI, Vasilyev IY, Boulygina EA, Ardatskaya MD, Pavlenko AV, Tyakht AV, Odintsova AK and Abdulkhakov RA: Markers of dysbiosis in patients with ulcerative colitis and Crohn's disease. Ter Arkh 91: 17-24, 2019.

55. Huttenhower C, Knight R, Brown CT, Caporaso JG, Clemente JC, Gevers D, Franzosa EA, Kelley ST, Knights D, Ley RE, et al: Advancing the microbiome research community. Cell 159: 227-230, 2014

56. Lynch SV and Pedersen O: The human intestinal microbiome in health and disease. N Engl J Med 375: 2369-2379, 2016

57. Daniels L, Budding AE, de Korte N, Eck A, Bogaards JA, Stockmann HB, Consten EC, Savelkoul PH and Boermeester MA: Fecal microbiome analysis as a diagnostic test for diverticulitis. Eur J Clin Microbiol Infect Dis 33: 1927-1936, 2014.
58. Hirano A, Umeno J, Okamoto Y, Shibata H, Ogura Y, Moriyama T, Torisu T, Fujioka S, Fuyuno Y, Kawarabayasi Y, et al: Comparison of the microbial community structure between inflamed and non-inflamed sites in patients with ulcerative colitis. J Gastroenterol Hepatol: Feb 20, 2018 (Epub ahead of print).

59. Pace F, Pace M and Quartarone G: Probiotics in digestive diseases: Focus on Lactobacillus GG. Minerva Gastroenterol Dietol 61: 273-292, 2015.

60. Takamura T, Harama D, Fukumoto S, Nakamura Y, Shimokawa N, Ishimaru K, Ikegami S, Makino S, Kitamura M and Nakao A: Lactobacillus bulgaricus OLL1181 activates the aryl hydrocarbon receptor pathway and inhibits colitis. Immunol Cell Biol 89: 817-822, 2011

61. Liu YW, Su YW, Ong WK, Cheng TH and Tsai YC: Oral administration of Lactobacillus plantarum K68 ameliorates DSS-induced ulcerative colitis in BALB/c mice via the anti-inflammatory and immunomodulatory activities. Int Immunopharmacol 11: 2159-2166, 2011.

62. Atkins HL, Geier MS, Prisciandaro LD, Pattanaik AK, Forder RE, Turner MS and Howarth GS: Effects of a Lactobacillus reuteri BR11 mutant deficient in the cystine-transport system in a rat model of inflammatory bowel disease. Dig Dis Sci 57: 713-719, 2012.

63. Clarke G, Sandhu KV, Griffin BT, Dinan TG, Cryan JF and Hyland NP: Gut reactions: Breaking down xenobiotic-microbiome interactions. Pharmacol Rev 71: 198-224, 2019.

64. Suez J, Zmora N, Segal E and Elinav E: The pros, cons, and many unknowns of probiotics. Nat Med 25: 716-729, 2019.

65. Meijer BJ and Dieleman LA: Probiotics in the treatment of human inflammatory bowel diseases: Update 2011. J Clin Gastroenterol 45 (Suppl 1): S139-S144, 2011.

66. Shi N, Li N, Duan X and Niu H: Interaction between the gut microbiome and mucosal immune system. Mil Med Res 4: 14, 2017.

67. Steck N, Hoffmann M, Sava IG, Kim SC, Hahne H, Tonkonogy SL, Mair K, Krueger D, Pruteanu M, Shanahan F, et al: Enterococcus faecalis metalloprotease compromises epithelial barrier and contributes to intestinal inflammation. Gastroenterology 141: 959-971, 2011.

68. Zhou Y, Chen H, He H, Du Y, Hu J, Li Y, Li Y, Zhou Y, Wang H, Chen Y and Nie Y: Increased Enterococcus faecalis infection is associated with clinically active Crohn disease. Medicine (Baltimore) 95: e5019, 2016.

69. Xie Z, Qu Y, Leng Y, Sun W, Ma S, Wei J, Hu J and Zhang $\mathrm{X}$ : Human colon carcinogenesis is associated with increased interleukin-17-driven inflammatory responses. Drug Des Devel Ther 9: 1679-1689, 2015.

70. D'Ambrosio A, Cossu A, Amendola A, Zandri A, Butera A, Sanchez M, Biffoni M, Pronio A, Montesani C, Kohn A, et al: Lamina propria $\mathrm{CD}^{+} \mathrm{LAP}^{+}$regulatory $\mathrm{T}$ cells are increased in active ulcerative colitis but show increased IL-17 expression and reduced suppressor activity. J Crohns Colitis 10: 346-353, 2016.

71. Zhu Q,Zheng P, Chen X,Zhou F, HeQ and Yang Y: Andrographolide presents therapeutic effect on ulcerative colitis through the inhibition of IL-23/IL-17 axis. Am J Transl Res 10: 465-473, 2018.

72. Kevans D, Tyler AD, Holm K, Jørgensen KK, Vatn MH, Karlsen TH, Kaplan GG, Eksteen B, Gevers D, Hov JR and Silverberg MS: Characterization of intestinal microbiota in ulcerative colitis patients with and without primary sclerosing cholangitis. J Crohns Colitis 10: 330-337, 2016.

73. Bajer L, Kverka M, Kostovcik M, Macinga P, Dvorak J, Stehlikova Z, Brezina J, Wohl P, Spicak J and Drastich P: Distinct gut microbiota profiles in patients with primary sclerosing cholangitis and ulcerative colitis. World J Gastroenterol 23: 4548-4558, 2017.

74. Rubio CA, Langner C and Schmidt PT: Partial to complete abrogation of the subepithelial macrophage barrier against the gut microbiota in patients with ulcerative colitis and Crohn's colitis. Histopathology 72: 580-587, 2018.

75. Kiely CJ, Pavli P and O'Brien CL: The role of inflammation in temporal shifts in the inflammatory bowel disease mucosal microbiome. Gut Microbes 9: 477-485, 2018

76. Yang $\mathrm{Y}$ and Jobin C: Novel insights into microbiome in colitis and colorectal cancer. Curr Opin Gastroenterol 33: 422-427, 2017.

77. Huttenhower C, Kostic AD and Xavier RJ: Inflammatory bowel disease as a model for translating the microbiome. Immunity 40 : 843-854, 2014

78. Slatko BE, Gardner AF and Ausubel FM: Overview of next-generation sequencing technologies. Curr Protoc Mol Biol 122: e59, 2018.

79. Eckburg PB, Bik EM, Bernstein CN, Purdom E, Dethlefsen L, Sargent M, Gill SR, Nelson KE and Relman DA: Diversity of the human intestinal microbial flora. Science 308: 1635-1638, 2005. 